\title{
Micromechanical fracture modeling of asphalt concrete using a single-edge notched beam test
}

\author{
Hyunwook Kim • Michael P. Wagoner • \\ William G. Buttlar
}

Received: 30 November 2007 / Accepted: 22 July 2008/Published online: 30 July 2008

(C) RILEM 2008

\begin{abstract}
Cracks in asphalt pavements create irreversible structural and functional deficiencies that increase maintenance costs and decrease lifespan. Therefore, it is important to understand the fracture behavior of asphalt mixtures, which consist of irregularly shaped and randomly oriented aggregate particles and mastic. A two-dimensional clustered discrete element modeling (DEM) approach is implemented to simulate the complex crack behavior observed during asphalt concrete fracture tests. A cohesive softening model (CSM) is adapted as an intrinsic constitutive law governing material separation in asphalt concrete. A homogenous model is employed to investigate the mode I fracture behavior of asphalt concrete using a single-edge notched beam (SE(B)) test. Heterogeneous morphological features are added to numerical SE(B) specimens to investigate complex fracture mechanisms in the process zone. Energy decomposition analyses are performed to gain insight towards the forms of energy
\end{abstract}

H. Kim $(\bowtie)$

Laboratory for Road Engineering/Sealing Components, Empa, Swiss Federal Laboratories for Materials Testing and Research, Überlandstrasse 129, 8600 Dübendorf, Switzerland

e-mail: hyunwook.kim@empa.ch

M. P. Wagoner · W. G. Buttlar

Department of Civil and Environmental Engineering, University of Illinois at Urbana-Champaign, Newmark Laboratory, 205 North Mathews Avenue, Urbana, IL 61801, USA dissipation present in fracture testing of asphalt concrete. Finally, a heterogeneous model is used to simulate mixed-mode crack propagation.

Keywords Discrete element method . Fracture $\cdot$ Cohesive softening model . Asphalt concrete $\cdot$ Heterogeneous

\section{Introduction}

The development of micromechanical models was initiated more than a hundred years ago. Micromechanical models that predict global material behavior based upon the properties of the individual constituents have been developed from 1880s. In general, micromechanical models can be grouped into two broad categories: non-interacting particles and interacting particles. As a further subclassification, non-interacting particle models can have specified geometries or non-specified geometries. Table 1 illustrates the model categories and applications to cementitious materials.

In asphalt concrete, there are a number of parameters that need to be considered in order to describe the system which contains one or more disperse phases embedded in a continuous matrix. These parameters include: (1) particle shape; (2) size and size distribution of particles; (3) concentration and concentration distribution of particles; (4) orientation of particles; (5) spatial distribution of particles; (6) 
Table 1 Evolution history of micromechanical models for fracture and non-fracture analyses of cementitious materials

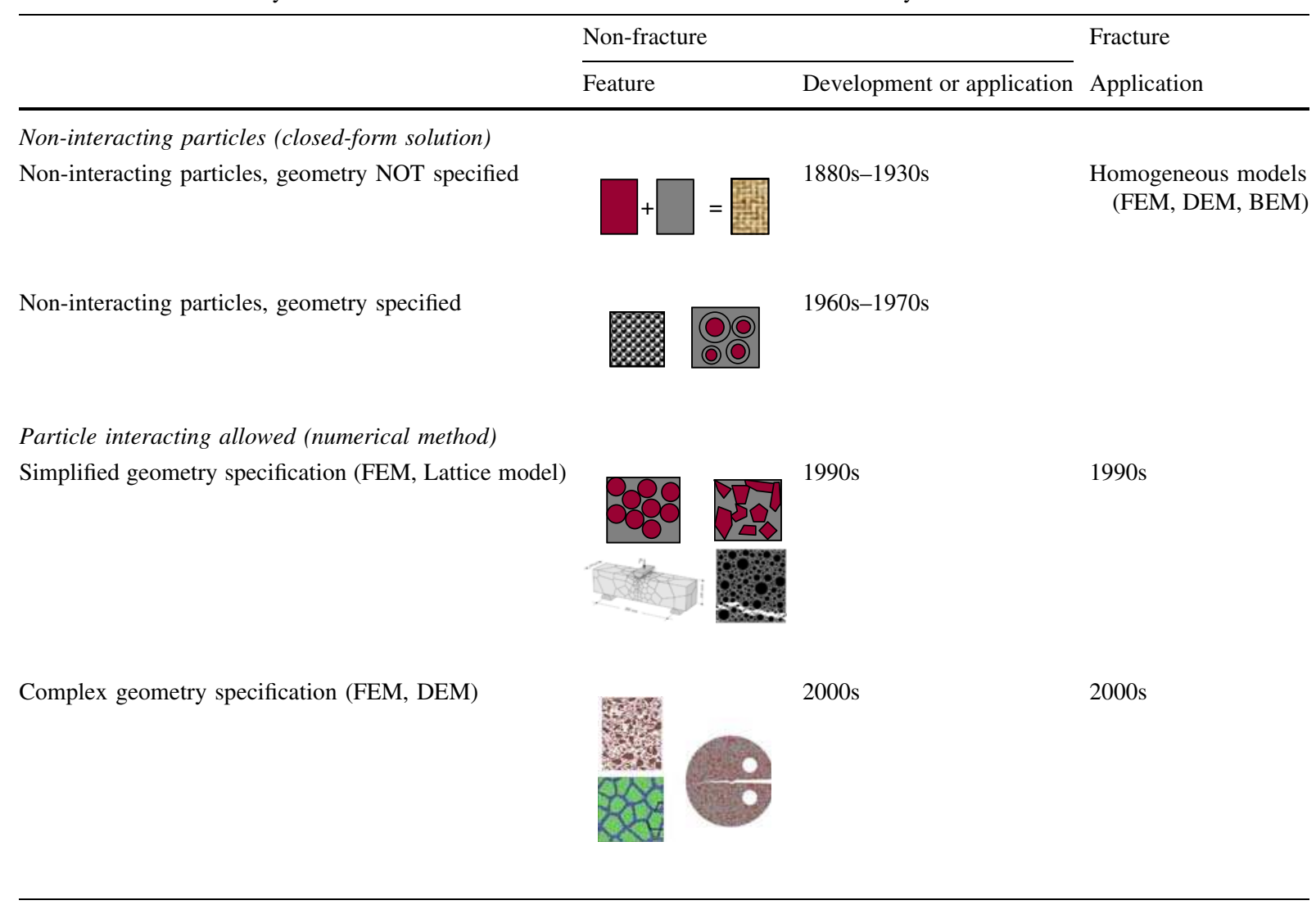

Particle interacting allowed (numerical method)

Simplified geometry specification (FEM, Lattice model)
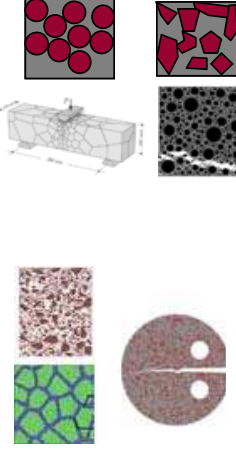

mogeneous models (FEM, DEM, BEM) 
Most studies in fracture of asphalt concrete have been limited to either experimental investigations or the study of stationary cracks. For more progressive crack propagation and numerical studies, homogeneous fracture models have been applied based on the cohesive zone model or Lattice model in conjunction with the continuum-based model [11, 12]. However, asphalt concrete is a quasi-brittle composite material which is composed of brittle aggregates and viscous mastic. The fracture of heterogeneous solids is a difficult problem to handle numerically due to the creation and continuous movement of new cracked surfaces. The use of nonlinear fracture mechanics to analytically describe these mechanisms can be extremely challenging, since the fracture patterns typically consist of a main crack, crack branches, secondary cracks and micro-cracks. Recently, more realistic fracture modeling with heterogeneous microstructure in asphalt concrete has been studied using advanced Lattice models and the discrete element method [13, 14].

The present study seeks to illustrate the potential usefulness of a new approach for studying crack behavior in asphalt concrete by directly accounting for the contribution of the material's heterogeneity by modeling discrete aggregate particles and the asphaltaggregate mastic. A clustered distinct element modeling approach was implemented in the twodimensional particle flow software package (PFC-2D) to study the complex crack behavior observed in asphalt concrete fracture tests [15]. In this work, an powerful integration of experimental test and numerical scheme, involving the cohesive softening model (CSM) and image analysis, is introduced to investigate the fracture behavior of asphalt concrete and to simulate crack nucleation, initiation and propagation including both mode I and mixed-mode behavior.

\section{Discrete element method}

The discrete element method (DEM) originally developed by Cundall [16] has proven to be a power and versatile numerical tool for modeling the behavior of granular and particulate systems, and also for studying the micromechanics of materials such as soil at the particle level. Also, the method has the potential to be an effective tool to model continuum problems, especially those that are characterized by a transformation from a continuum to discontinuum. The DEM discretizes a material using rigid elements of simple shape that interact with neighboring elements according to interaction laws that are applied at points of contact.

The analysis procedure consists of three major computational steps: internal force evaluation, in which contact forces are calculated; integration of equations of motion, in which element displacement are computed; and contact detection, where new contacts are identified and broken contacts are removed. In a discrete element analysis, the interaction of the elements is treated as a dynamic process that alternates between the application of Newton's second law and the evaluation of a force-displacement law at the contacts. Newton's second law gives the acceleration of an element resulting from the force acting on it, including gravitational forces, external forces prescribed by boundary conditions, and internal forces developed at inter-element contacts. The acceleration is then integrated to obtain the velocity and displacement. The force-displacement law is used to find contact forces from known displacement. The equations of motion are integrated in time using the central difference method. Details of this process are given in a paper by Cundall [16]. The method can be computationally very demanding and thus, efficient algorithms, especially for the internal force evaluations and contact detection, must be used. Computational effectiveness will be particularly important for three-dimensional discretizations, the use of which is inevitable for obtaining fully realistic and accurate models for many applications.

In the absence of damping, the DEM equilibrium equation at discrete time intervals for the system of particles is following:

$M a+K \Delta x=\Delta f$

where, $M$ is the mass matrix, $a$ is the acceleration vector, $K$ is the stiffness matrix, $\Delta f$ is the incremental force vector, and $\Delta x$ is the incremental displacement vector. The translational and rotational stiffnesses of a particle relate increments of force and moment to increments of displacement and rotation via the matrix relations:

$\{\Delta f\}=[K]\{\delta u\}$

In a two-dimensional system, Eq. 2 can be expressed as: 


$$
\left\{\begin{array}{l}
\Delta F_{1} \\
\Delta F_{2} \\
\Delta M_{3}
\end{array}\right\}=\left[\begin{array}{ccc}
k_{11} & k_{12} & 0 \\
k_{21} & k_{22} & 0 \\
0 & 0 & k_{33}
\end{array}\right]\left\{\begin{array}{c}
\Delta U_{1} \\
\Delta U_{2} \\
\Delta \theta_{3}
\end{array}\right\}
$$

The individual elements of this stiffness matrix can be expressed for a particular contact in terms of the particle radius $R$, the contact normal vector $n_{i}$, and the contact stiffnesses, i.e. $K_{n}$ and $K_{s}$. Again, Eq. 2 can be expressed with the terms in a standard FE frame work from Eq. 4 to Eq. 7:

$$
\begin{aligned}
& \{\Delta f\}=\left(\int[B]^{T}[D][B] d V\right)\{\delta u\} \\
& \{\varepsilon\}=[B]\{\delta u\} \\
& \{\sigma\}=[D]\{\varepsilon\}=[D][B]\{\delta u\} \equiv \Delta \sigma_{1} \\
& \{\Delta f\}=\int[B]^{T} \Delta \sigma_{1} d V
\end{aligned}
$$

where, $\{\Delta f\}$ are the incremental force vectors, $[K]$ is the contact stiffness matrix, $\{\delta u\}$ are the contact displacements, $\{\varepsilon\}$ are the contact strains, $\{\sigma\}$ are the contact stresses, $[B]$ is the strain matrix and $[D]$ is the elasticity matrix.

The elements of the stiffness matrix represent primarily the normal and shear springs that are present at the contact points. The stiffness matrix $([K])$ changes during the analysis as contacts are formed and broken. Discrete element simulations can therefore be classified as non-linear, dynamic analyses. The principal difference between the available DEMs is the time integration algorithm used to solve Eq. 1 [17]. Equation 2 is similar to the global equation considered in continuum finite element modeling. An analogy can therefore be drawn between a discrete element framework and a finite element framework; discrete element particles corresponding to the finite element nodes and inter-particle contacts corresponding to finite element as shown in Fig. 1.

The constitutive models used in the current 2-D discrete element model application consist of three parts: a contact stiffness model, a slip friction model and a bonding model. The linear contact model is defined by the normal and shear stiffness, $K_{n}$ and $K_{s}$ (force/displacement), of the two contacting entities (ball-to-ball or ball-to-wall) acting in series. The details of the basic constitutive laws used to model bulk material response (normal and shear stiffness and frictional sliding) can be found in the PFC manual.

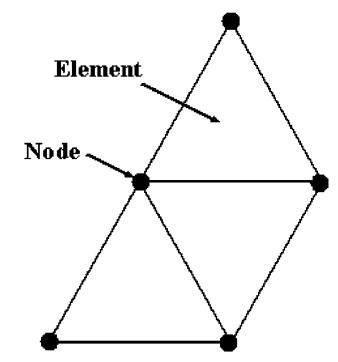

(a)

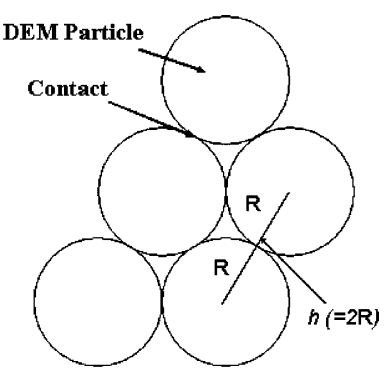

(b)
Fig. 1 Schematic diagram illustrating DEM-FEM analogy. (a) Elements and nodes (FEM) and (b) particles and contacts (DEM)

\section{Cohesive fracture model}

Dugdale [18] and Barenblatt [19] proposed cohesive models to investigate ductile and brittle material fracture behavior, respectively. The cohesive crack concept was later extended by Hillerborg et al. [20] to study nonlinear fracture processes in Portland cement concrete. Furthermore, cohesive zone models (CZMs) have been used to simulate the fracture process in a number of material systems including polymers, metallic materials, ceramic materials, metal matrix composites, and fiber reinforced plastic composites under varying loading conditions, e.g. static, dynamic, and cyclic. The first application of a cohesive zone model to simulate asphalt materials was made by Jenq and Perng [11] although material microstructure was not considered in their study. Discrete element models can also be used to simulate material fracture by utilizing more sophisticated bonding models, including softening-type constitutive models. These tools can be used to simulate crack initiation and propagation in a manner similar to the cohesive zone modeling approach as shown in Fig. 2. The cohesive crack models provide the capability to simulate the crack propagation occurring in a process zone located ahead of a crack tip. The cohesive approaches involve nonlinear constitutive laws described by the displacement jump and the corresponding traction along the contact interfaces. The area under this local cohesive model curve is a fracture energy, which is needed for creating the crack surface. As shown in Fig. 2, the initial slope of local cohesive softening model represents material modulus. The material strength can be determined by 


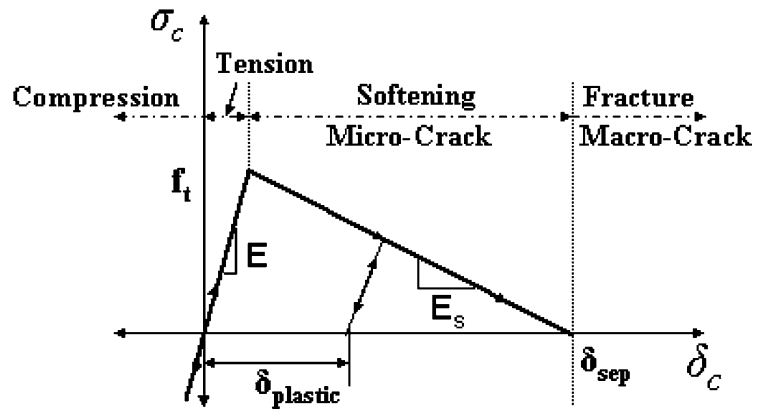

Fig. 2 Concept of cohesive softening model

the radius of particle and the thickness in a twodimensional discrete element model [21].

The softening slope is varied with the distance of the center locations of adjacent particles $(h)$, as shown in Fig. 1, in order to maintain a constant fracture energy dissipation rate and ensure the mesh objectivity as shown Eq. 8 [22].

$E_{s}=\frac{1}{\left[\left(\frac{2 G_{f}}{f_{t}^{2} h}\right)-\frac{1}{E}\right]}$

where, $E_{s}$ is the softening modulus, $G_{f}$ is the cohesive fracture energy dissipation rate.

Cohesive softening models are described by three parameters (two are independent), namely the cohesive fracture energy dissipation rate, $G_{f}$, the cohesive strength, $f_{t}$, and the separation/critical length, $\delta_{\text {sep }}$. In general, cohesive energy is obtained from experiments and is generally assumed to be equivalent to the work of fracture $\Gamma$. The cohesive energy potential is simply:

$G_{f}=\int_{0}^{\delta_{\text {sep }}} \sigma_{c}(\delta) d \delta$

The verification of cohesive fracture model was already conducted using a double cantilever beam and the results were almost identical to the analytical solution $[14,23]$.

\section{Governing equation of energy determination}

For more detail fracture analysis, the energy flow of micromechanical facture model needs to be calculated. The energy in the entire particle assembly can be tracked with respect to the following five partitions. They are boundary, strain, friction, kinetic, and fracture energy.

\subsection{Boundary energy}

Boundary energy is total accumulated work, $E_{w}$, done by all walls, which were used for applying the external load, on the assembly.

$E_{W}=E_{W}-\sum_{N_{W}}\left(F_{i} \Delta U_{i}+M_{3} \Delta \theta_{3}\right)$

where, $N_{w}$ is the number of walls; $F_{i}$ and $M_{3}$ are the resultant force and moment acting on the wall at the start of the current time step; and $\Delta U_{i}$ and $\Delta \theta_{3}$ are the applied displacement and rotation occurring during the current time step. Also note that $E_{w}$ may be positive or negative, with the convention that work done by the walls on the particles is positive.

\subsection{Strain energy}

Strain energy is the total accumulated strain work done across the entire assembly.

$E_{S}=\frac{1}{2} \sum_{N_{C}}\left(\left|F_{i}^{n}\right|^{2} / K_{n}+\left|F_{i}^{s}\right|^{2} / K_{s}\right)$

where, $N_{c}$ is the number of contacts; $\left|F_{i}^{n}\right|$ and $\left|F_{i}^{s}\right|$ are the magnitudes of the normal and shear components of the contact force. In the case of the bulk material behavior, this energy is stored and can be recovered upon unloading. This will be apparent when we examine the decomposition of energy as the crack progresses through the specimen in a later section. As the specimen becomes fully cracked, resembling a hinged, two-piece structure, the bending of the bulk material becomes negligible as evidenced by the reduction in stored strain energy.

\subsection{Frictional energy}

Frictional energy is total energy, $E_{f}$, dissipated by frictional sliding at all contacts:

$E_{f}=E_{f}-\sum_{N_{C}}\left(\left\langle F_{i}^{s}\right\rangle\left(\Delta U_{i}^{s}\right)^{s l i p}\right)$

where, $N_{c}$ is the number of contacts; $\left\langle F_{i}^{s}\right\rangle$ and $\left(\Delta U_{i}^{s}\right)^{\text {slip }}$ are the average shear force and the increment of slip displacement, respectively, at the contact for the current time step. 


\subsection{Kinetic energy}

Kinetic energy is total kinetic energy, $E_{k}$, of all particles accounting for both translational and rotational motion. $E_{k}$ can be expressed in terms of the generalized mass, $M_{(i)}$, and velocity, $V_{(i)}$, of each of the $N_{p}$ particles as:

$E_{k}=\frac{1}{2} \sum_{N_{p}} \sum_{i=1}^{3} M_{(i)} V_{(i)}^{2}$

\subsection{Fracture energy}

Fracture energy can be determined by subtracting all other energies from the total (or boundary) energy easily.

$\Gamma_{c}=E_{w}-E_{s}-E_{f}-E_{k}$

\section{Single-edge notched beam test}

The selection of the specimen geometry was the first step in developing a fracture test for asphalt concrete to use for integrating with numerical models. Several parameters were defined when selecting the specimen geometry, such as test simplicity (specimen fabrication, test fixtures, etc.), amenable stress states (simple stress fields, minimal end effects), and the ability to obtain fracture energy. The single edge notched beam (SE(B)) geometry (Fig. 3) can be readily fabricated from laboratory compacted beams or slabs. The loading configuration (three-point bend) allows for simple stress states and ease of test control with

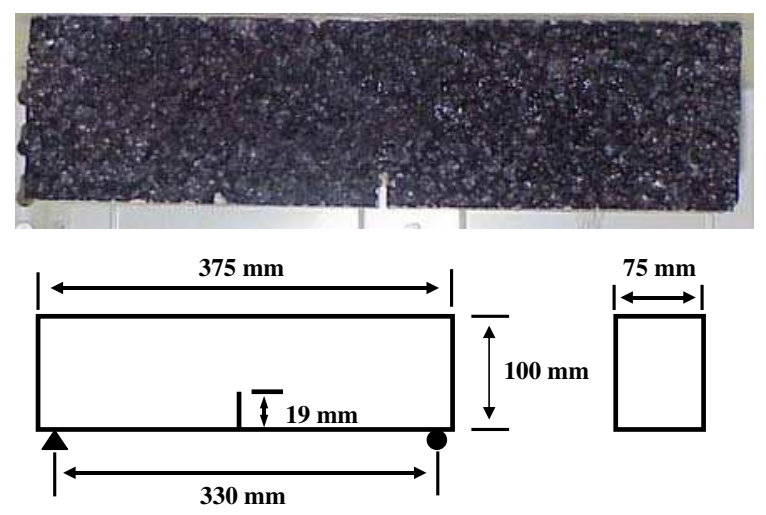

Fig. $3 \mathrm{SE}(\mathrm{B})$ Geometry and dimensions for asphalt concrete

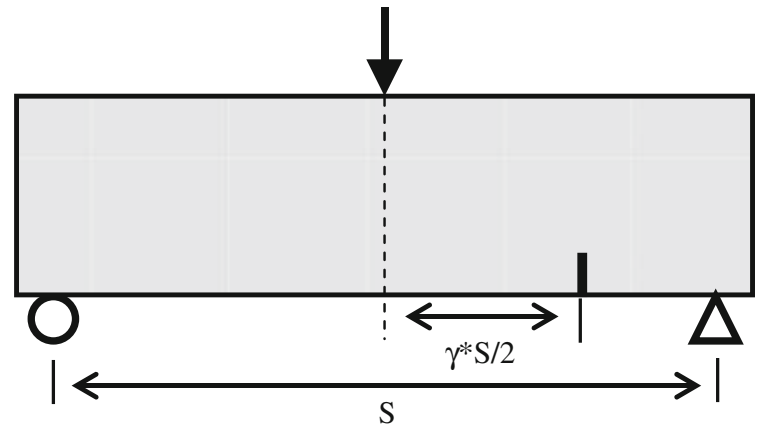

Fig. 4 Mixed-mode test geometry using offset notch method

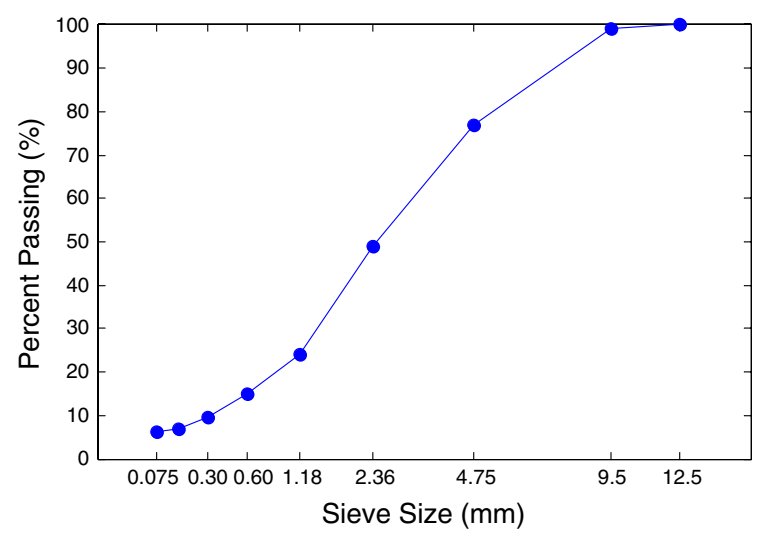

Fig. 5 Gradation plot of asphalt mixture

closed-loop servo-hydraulic equipment. Moreover, by simply offsetting the mechanical notch location (see Fig. 4), the notched beam test can be used to characterize mixed-mode fracture characteristics. Further details of the notched beam fracture test for asphalt concrete can be found in an author's publication [24].

The asphalt concrete mixture utilized for this study is a typical central Illinois surface mixture that consists of a $9.5 \mathrm{~mm}$ nominal maximum aggregate size and a PG (Performance Grade) 64-22 asphalt binder. The gradation plot for the mixture is shown in Fig. 5. This particular mixture has been extensively characterized by Wagoner [25] including the bulk material properties required for the material inputs for the numerical model.

\section{Mode I fracture simulations}

\subsection{Geometry of numerical model}

Figure 6 illustrates a simply-supported, single-ended notched beam with a length of $376 \mathrm{~mm}$, a height of 
Fig. 6 Mode I DEM fracture model geometry and mesh

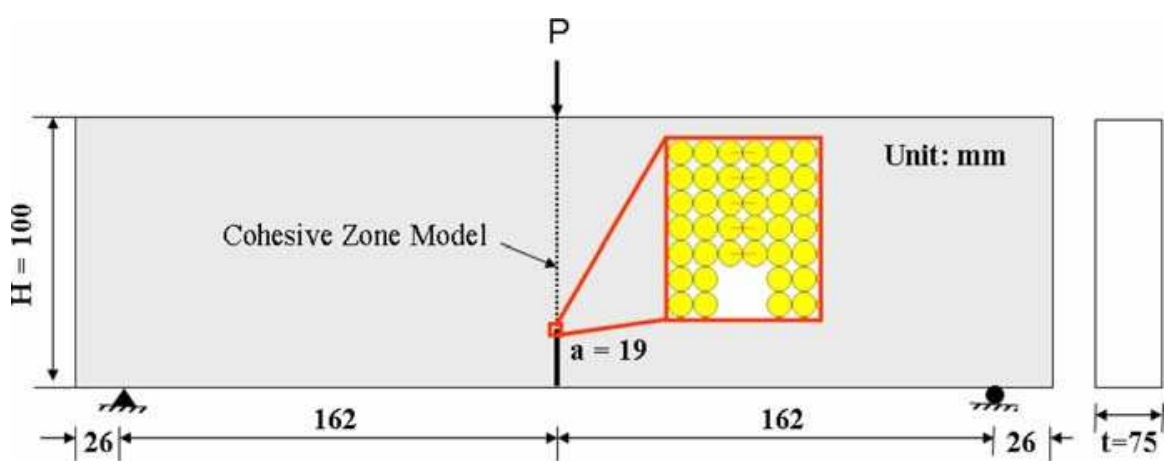

$100 \mathrm{~mm}$ and a thickness of $75 \mathrm{~mm}$. A mechanical notch was then inserted with a length of $19 \mathrm{~mm}$, giving a notch to depth $(a / H)$ ratio of 0.19 . The depth of the notch was selected to be long enough to ensure adequate stress intensity at the notch tip to initiate a crack, but short enough to ensure a ligament of adequate length for test repeatability and to prevent crack initiation under self-weight. Constant velocity (or displacement) boundary conditions are imposed at the center of the top edge of the model in order to predict stable fracture behavior. Figure 6 shows a discrete element configuration for the small region around crack tip along the middle of the specimen respectively. Two-dimensional cohesive contact models are inserted only along the center of the specimen. The bulk material is modeled as elastic, homogeneous, and isotropic with a face-centered arrangement.

\subsection{Material parameters}

Two experimental fracture properties, material strength and fracture energy, are evaluated herein as material inputs into the cohesive fracture model, which can be calibrated. The first-failure tensile strength determined from the indirect tension test at $-10^{\circ} \mathrm{C}$ and $1 \mathrm{~Hz}$ is defined as the material strength. The procedure for determining the first-failure tensile strength is outlined in the AASHTO T322-03 specification [26]. The material tensile strength of 3.56 MPa was obtained in the IDT test for the dense-graded limestone-dolomite asphalt mixture studied herein. The notched beam fracture test was used for determining the fracture energy of the mixture under investigation. In this experiment, the crack mouth opening displacement (CMOD) was increased at a linear rate. The fracture energy was then determined by calculating the area under the load-displacement curve and normalizing by the cross-sectional area of the beam. A fracture energy of $344 \mathrm{~J} / \mathrm{m}^{2}$ was obtained from the notched beam test. Because the fracture energy obtained in this method currently overestimates the energy associated with the material separation, it is convenient and logical to adjust this parameter for model calibration.

\subsection{Homogeneous fracture analysis}

For the homogenous model, 37,981 particles with $0.5 \mathrm{~mm}$ radius and 75,442 contacts are used across the entire specimen, while 81 cohesive contacts are inserted along the middle of specimen. Young's modulus of 14.2 GPa was assigned for bulk material and the calibrated strength for cohesive fracture model was 3.21 MPa. The calibrated fracture energy was $241 \mathrm{~J} / \mathrm{m}^{2}$. A parametric study was conducted to determine the sensitivity of the particle size on the numerical results. Three different particle radii $(0.25 \mathrm{~mm}, 0.5 \mathrm{~mm}$ and $1.0 \mathrm{~mm})$ were utilized in the simulations. The result demonstrates that within the selected size range used in the fracture study, global fracture response is independent of particle size [27]. However, the selection of different particle size in heterogeneous fracture model will affect to the distribution of microcracks so it is an important aspect in the applications of heterogeneous fracture models [28]. Figure 7 represents the experimental and numerical global results as expressed in terms of force versus CMOD with asphalt mixtures with $9.5 \mathrm{~mm}$ nominal maximum aggregate size (NMAS). In the calibrated model, the force versus CMOD curve was quite reasonable as compared to experimental results, which is not surprising, since nonlocal material properties were used as inputs to the 


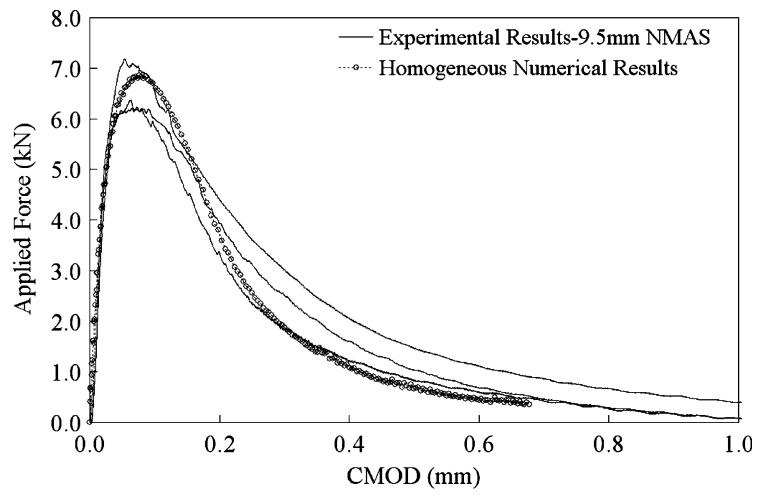

Fig. 7 Experimental and homogeneous numerical results $\left(9.5 \mathrm{~mm}\right.$ NMAS at $\left.-10^{\circ} \mathrm{C}\right)$

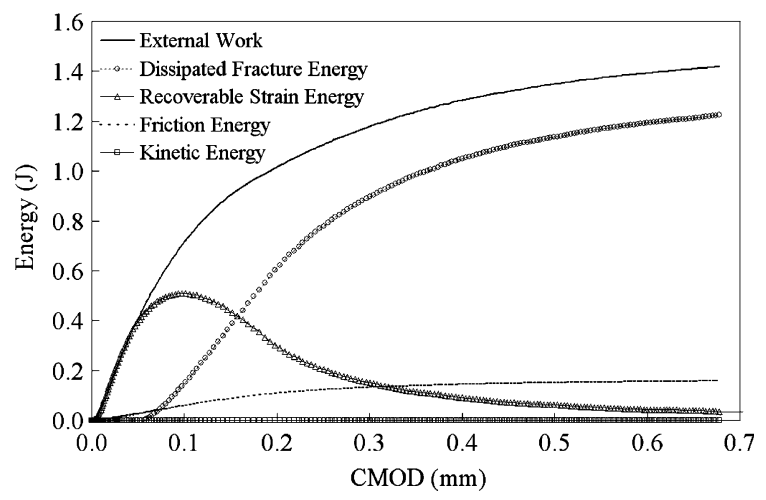

Fig. 8 Energy balance of homogeneous DEM fracture model

intrinsic cohesive softening model as a first-order approximation (e.g. the tensile strength obtained from the indirect tensile test and the fracture energy from the notched beam test).

Figure 8 shows a decomposition of energy storage and dissipation, which indicates that strain, fracture, and friction energies are dominant in the fracture test, while kinetic energy is essentially negligible. The strain energy has the highest value when the applied force reaches the peak load. After the peak point, the stored strain energy in the specimen decreases as the crack propagates and the energy becomes highly localized in the remaining hinge-like ligament ahead of the crack.

\subsection{Heterogeneous fracture analysis}

With high resolution optical image equipment and powerful image processing techniques, a heterogeneous discrete element fracture model can be constructed. The realistic microstructure can be obtained and projected onto the discrete element mesh using Image-Pro Plus [29] and a user-defined visual basic program code. The detail analysis procedure of image processing will not be discussed in this paper due to the limitation of paper length. However, it can be found in other publication [27].

Figure 9 represents the numerical heterogeneous model geometry and crack propagation results. In this model, 149,922 particles with $0.25 \mathrm{~mm}$ radius and 298,855 contacts are used across the entire specimen and 61,717 particles and 105,236 contacts are used to model aggregates; 88,205 particles and 158,042 contacts for mastic, and; 35,577 contacts are used at the interfaces between aggregate and mastic sub-particles. Detailed model parameters used in the simulations are provided in Table 2. The material parameters of aggregate and mastic were obtained from indirect tension and dynamic modulus tests [30]. The material parameters of interface were assumed that they are similar to the mastic but they should be continuously verified by advanced tests and measurements in future [31]. The fracture energies of mastic and interface were estimated from the inverse analysis and calibrated based on the experimental fracture test data of mixture.

The stress concentration in the ligament along the expected mode I crack path is significant and many micro-cracks were predicted in the process zone prior to crack propagation, as shown in Fig. 9b. Figure 9a and $\mathrm{b}$ show many micro-cracks around an aggregate in the top of notched crack tip. From the experimental tests and numerical simulations, the powerful potential benefits of discrete fracture model can be discussed. The fracture of asphalt concrete has very different fracture mechanisms due to the non-homogeneity in the specimen such as micro-cracking, crack branching and deflection, crack face sliding, crack bridging, and crack tip blunting. The microcracking phenomenon consumes a part of the external energy caused by the applied load. Crack deflection occurs when the path of least resistance is around a relatively strong particle or along a weak interface. Also, during the opening of a tortuous crack, there must be some frictional sliding between the cracked faces that causes energy dissipation through friction. Based on these mechanisms in the fracture of asphalt concrete, heterogeneous DEM fracture model can have the ability to gain some insight towards fracture toughening mechanisms in asphalt concrete. 
Fig. 9 Heterogeneous DEM fracture model and representations of cracks. (a) Numerical SE(B) specimen with microstructure, (b) microcracks and (c) macro-cracks

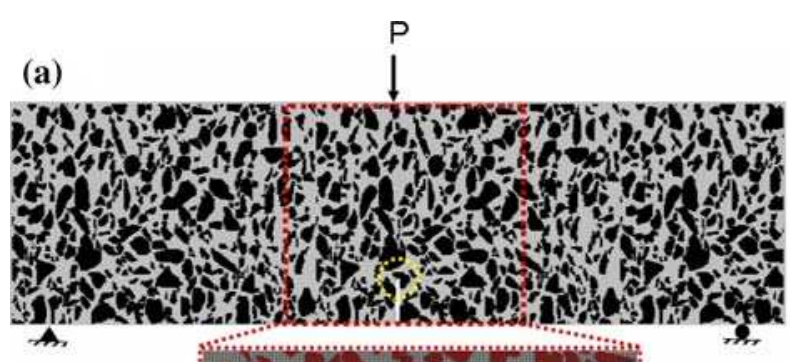

(b)

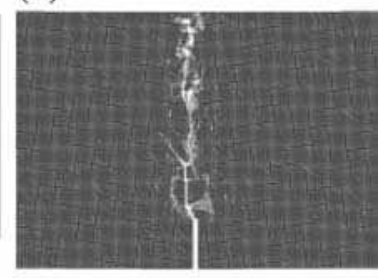

(c)

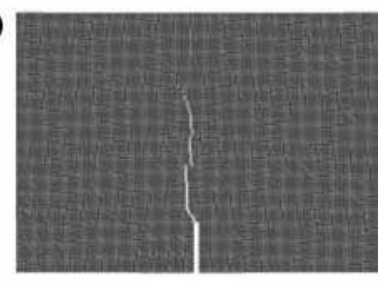

Table 2 Parameters for heterogeneous DEM fracture model $\left(9.5 \mathrm{~mm}\right.$ NMAS at $\left.-10^{\circ} \mathrm{C}\right)$

\begin{tabular}{|c|c|c|c|c|c|}
\hline \multirow{2}{*}{$\begin{array}{l}\text { SE(B) } \\
\text { Phase }\end{array}$} & \multicolumn{2}{|l|}{ Material properties } & \multicolumn{3}{|c|}{ DEM contact properties } \\
\hline & Young's modulus (GPa) & Strength $(\mathrm{MPa})$ & Stiffness $(\mathrm{GPa})$ & Bond force $(\mathrm{N})$ & Separation displacement (m) \\
\hline Aggregate & 56.8 & 6.59 & 4.26 & 247.2 & 7.6E-8 \\
\hline Mastic & 18.2 & 3.78 & 1.36 & 141.75 & $1.4 \mathrm{E}-4$ \\
\hline Interface & 18.2 & 3.44 & 1.36 & 136.18 & $4.5 \mathrm{E}-5$ \\
\hline
\end{tabular}

For the calibrated heterogeneous fracture simulation, the overall trend resembled the experimental result as shown in Fig. 10. Due to the presence of a large aggregate just ahead of the simulated notch, the force was predicted to experience a sudden decrease before the peak load but then was recovered. This phenomenon has been observed in experimental trial,

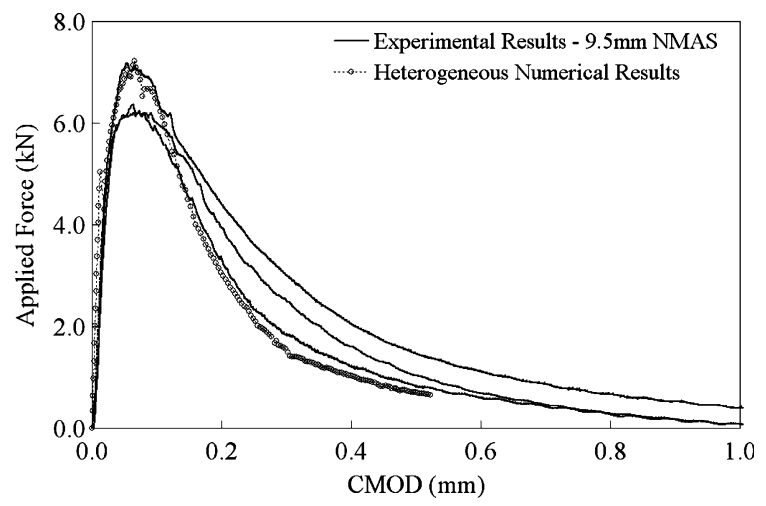

Fig. 10 Experimental and heterogeneous numerical results (9.5 mm NMAS at $\left.-10^{\circ} \mathrm{C}\right)$ particularly when thin specimens with larger aggregates are tested. Thus, the 2-D model herein tends to exaggerate the effect of aggregates on the loaddisplacement response, especially when compared to experimental results obtained from specimens with larger thicknesses-to-maximum-aggregate size ratios. Thus, the need for three-dimensional modeling to accurately capture material heterogeneity is apparent.

The strain and fracture energy in Fig. 11 also shows the aggregate effect in the sudden jump in the energy traces. A more detailed study of the fracture zone size and fracture toughening mechanisms is the focus of ongoing work. The difference in energy decomposition in the homogeneous and heterogeneous simulations was due to assumed material properties for the heterogeneous simulation. Like the results of homogeneous fracture model, the stored strain energy in the specimen decreases as the crack propagates. The friction energy is varied based on the friction coefficient of materials as well as the heterogeneity through the crack path. In this simulation, the friction coefficient of 0.5 was used for all materials. 


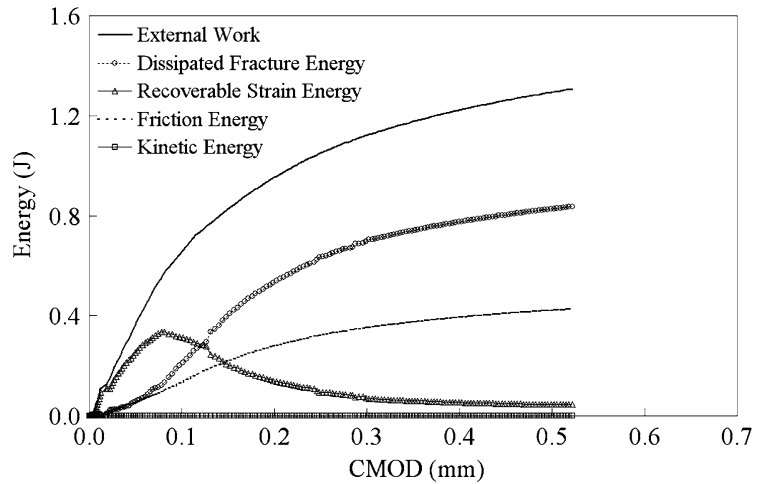

Fig. 11 Energy balance of heterogeneous DEM fracture model

\section{Mixed-mode fracture simulations}

The mixed-mode fracture in a pavement system is popular and significant due to the presence of continuous dynamic and static traffic loads and environmental conditions including temperature and moisture. Using the calibrated cohesive parameters and particle size $0.25 \mathrm{~mm}$, the mixed-mode problem was investigated herein using the beam fracture test with an offset notch to create mixed-mode (i.e. mixed with Mode-I and Mode-II) crack propagation. The offset parameters, $\gamma$, as defined in Fig. 12a, were 0.4,
0.5 , and 0.55 resulting in offset lengths of $65 \mathrm{~mm}$, $81 \mathrm{~mm}$, and $89 \mathrm{~mm}$ from the middle of specimen.

Figure $12 \mathrm{~b}$ presents the nomenclature used to be described the components of crack displacement around a notch with a propagating crack in the middle of specimen. The global displacements describing material separation are the crack mouth displacement (CMD), crack mouth opening displacement (CMOD), and crack mouth sliding displacement (CMSD). The more localized displacements, which are more closely tied to crack tip properties, are the crack tip displacement (CTD), crack tip opening displacement (CTOD), and crack tip sliding displacement (CTSD). These are important response indicators that can be obtained experimentally (with some difficulty) and/or estimated though numerical simulation. These parameters are important in model calibration and validation.

Figure 13 illustrates the crack paths from several beams tested with different offset lengths. The stress state within the mixed-mode beams are such that as the offset length increases, the shear component increases while the tensile component decreases. The experimental results show that the critical offset length for this geometry and mixture is between $\gamma=0.5$ and 0.55 . The critical offset length is defined as the length where the crack initiates and propagates
Fig. 12 Mixed-mode SE(B) geometry with offset notch. (a) Geometry of mixed-mode SE(B) and (b) composition of crack tip displacement and crack mouth displacement

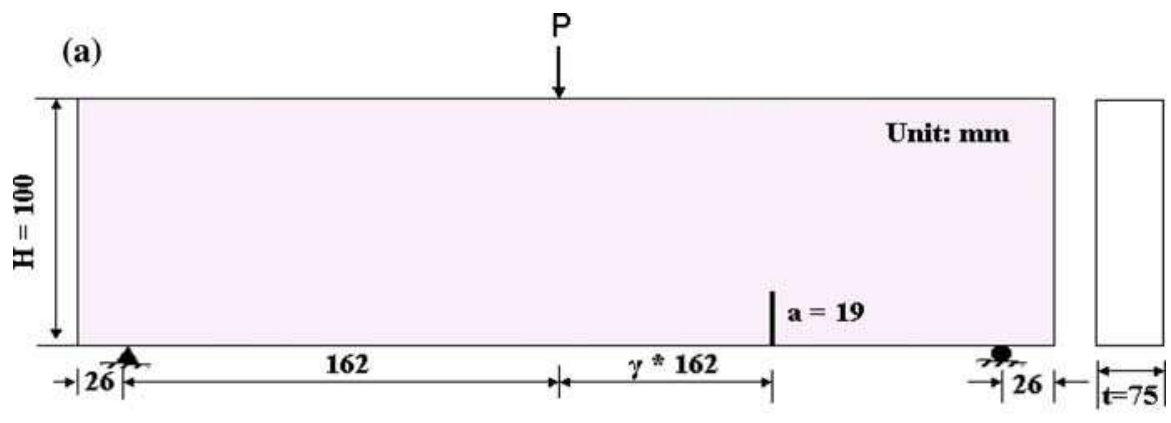

(b)

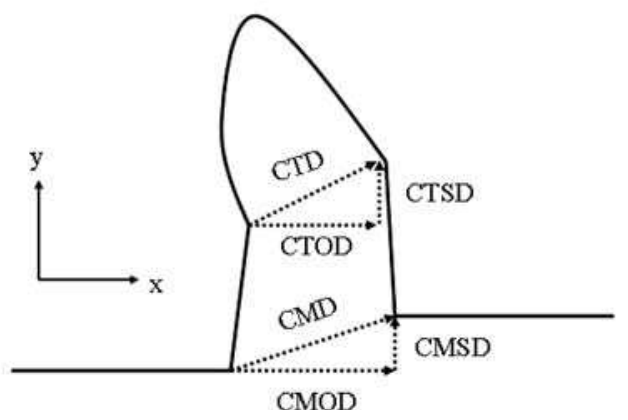




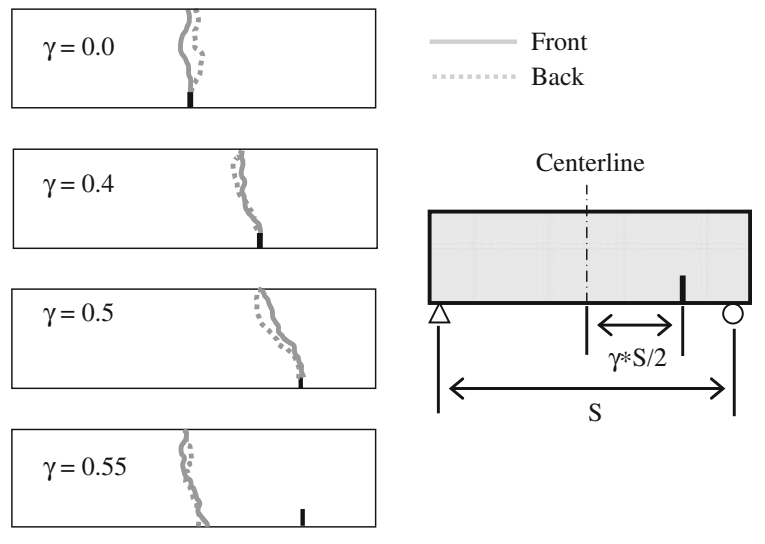

Fig. 13 Experimental crack path with various notch offsets

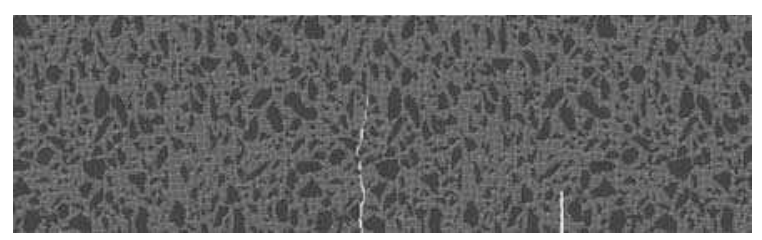

Fig. 14 Numerical crack trajectories trajectory $(\gamma=0.55)$

the centerline as opposed to the notch tip. The reason for the difference in initiation location is that the stress intensity at the notch tip reduces as the offset length increases and the tensile stress at the bottom centerline of the beam increases. The critical offset length is dependent on the geometry of the beam, i.e. span length and notch length. Figure 14 shows the crack trajectory of heterogeneous fracture model with 0.55 offset. As shown Fig. 13, both numerical and experimental crack trajectories are matched well for the crack propagation although the crack path is not exactly the same with experimental crack path.

The numerical crack trajectories with different notch offsets $(\gamma=0.4$ and 0.55$)$ were shown with the micro-crack and macro-crack distribution both locations of notched cracks in Fig. 15. The paths of crack propagations by fracture models were the same with the experimental test results. Competitions exist as to where more critical conditions prevail that will lead to macro-crack. The distributions of microcracks will be dependent on the heterogeneity around the critical locations and the features of offset notch. Furthermore, the density of micro-cracks generally decreases with increasing distance from the face of the main crack.

For the beam with 0.4 notch offset, the macrocrack initiated with mode-I fracturing in but the path quickly began to meander at an angle towards the loading point as the crack propagated. Before crack initiation, inelastic effects at the crack tip in the notched region and in the vicinity of the outer fiber of bending at the bottom of the mid-span of specimen are simultaneously occurring (Fig. 16).

\section{Summary and conclusions}

A numerical fracture model was constructed based on the cohesive softening model and a image processing technique for obtaining the microstructure of asphalt concrete specimen. Material parameters of numerical fracture model were determined from experimental tests. Using inverse analysis, the multi-phase material properties were obtained and applied into discrete element fracture models. Both mode-I and mixedmode fracture behaviors based on notched beam tests were investigated with the integration of experimental test and numerical simulations. From heterogeneous fracture models, the crack initiation and propagation could be predicted well and it was found that the solid aggregate can be broken at low temperatures if it is along the fracture process zone. Also, macro- and micro-crack distribution could be represented by a
Fig. 15 Numerical crack distributions with microand macro-cracks. (a) Crack distribution $(\gamma=0.4)$ and (b) crack distribution $(\gamma=0.55)$
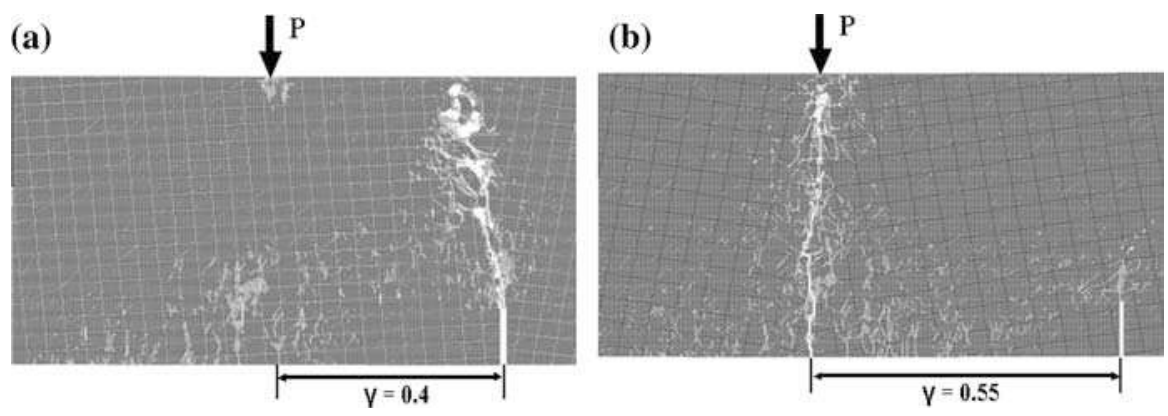

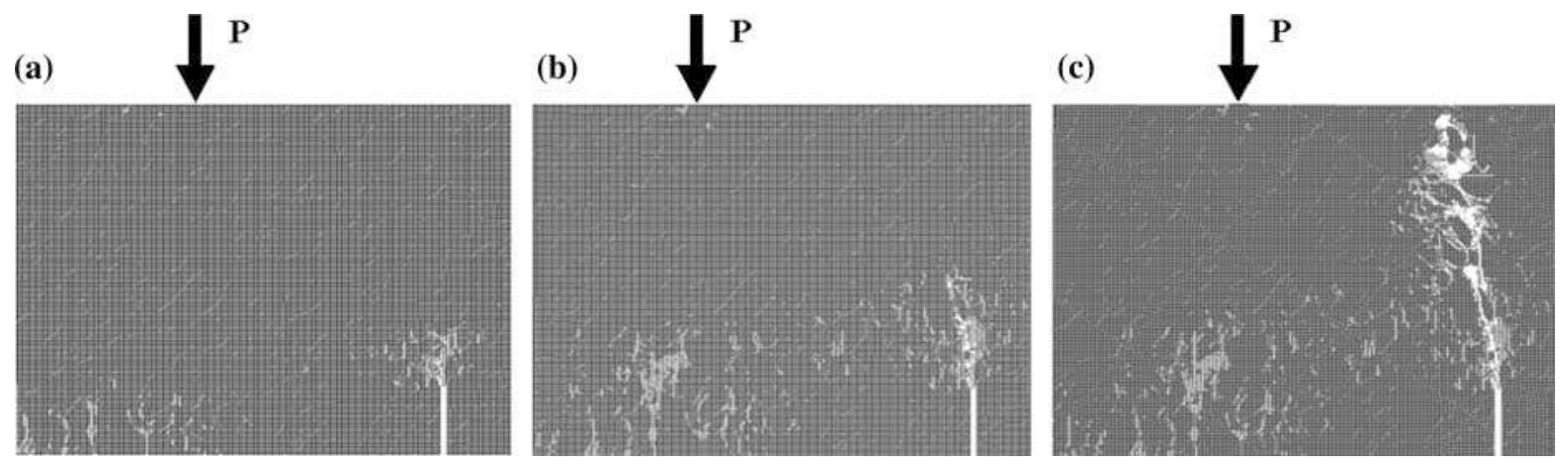

Fig. 16 Progressive crack initiation and propagation by numerical model $(\gamma=0.4)$. (a) Micro-cracks initiation, (b) macro-crack initiation and (c) crack propagation

numerical tool and the crack competition between critical tensile locations could be predicted in the mixed-mode fracture simulation. Energy decompositions were accomplished from homogeneous and heterogeneous fracture models but need to be validated with robust measurement tools. The energy analysis should ultimately be extended to include viscoelastic material response in conjunction with material fracture. Although much more works such as the interface fracture test and measurement of micro-cracks are needed to validate the numerical fracture models presented herein, discrete element fracture modeling approach appears to have significant potentials for aiding in the understanding of fracture behavior in asphalt concrete.

\section{References}

1. Buttlar WG, Roque R (1996) Evaluation of empirical and theoretical models to determine asphalt mixture stiffnesses at low temperatures. J Assoc Asphalt Paving Technol 65:99-141

2. Sadd MH, Dai Q, Parameswaran V, Shukla A (2004) Microstructural simulation of asphalt materials: modeling and experimental studies. ASCE J Mater Civ Eng 16:107-115

3. Chang CS, Wang TK, Sluys LJ, van Mier JGM (2002) Fracture modeling using a micro-structural mechanics approach-I. Theory and formulation. Eng Fract Mech 69:1941-1958

4. D'Addetta GA (2004) Discrete models for cohesive frictional materials. Report No. 42, Institute of Structural Mechanics, University of Stuttgart, Germany

5. Bazant ZP, Planas J (1998) Fracture and size effect in concrete and other quasibrittle materials. CRC Press, New York

6. Schlangen E, van Mier JGM (1992) Experimental and numerical analysis of the micro-mechanisms of fracture of cement-based composites. J Cem Concr Compos 14(2):105-118

7. Majidzadeh K, Kauffmann EM, Ramsamooj DV (1971) Application of fracture mechanics in the analysis of pavement fatigue. J Assoc Asphalt Paving Technol 40:227-246

8. Abdulshafi AA, Majidzadeh K (1985) J-integral and cyclic plasticity approach to fatigue and fracture of asphalt mixes. J Transp Res Rec 1034:112-123

9. Kim KW, El Hussein HM (1995) Effect of differential thermal contraction on fracture properties of asphalt materials at low temperature. J Assoc Asphalt Paving Technol 64:474-499

10. Jacob MM, Hopman PC, Molenaar AAA (1996) Application of fracture mechanics in principles to analyze cracking in asphalt concrete. J Assoc Asphalt Paving Technol 65:1-39

11. Jenq Y, Perng J (1991) Analysis of crack propagation in asphalt concrete using cohesive crack model. J Transp Res Rec 1317:90-99

12. Paulino GH, Song SH, Buttlar WG (2004) Cohesive zone modeling of fracture in asphalt concrete. In: Proceeding of fifth RILEM international conference on pavement cracking, Limoges, France

13. Chang KG, Meegoda JN (1997) Micromechanical simulation of hot mix asphalt. J Eng Mech 123(5):495-503

14. Kim H, Buttlar WG (2005) Micromechanical fracture modeling of hot-mix asphalt concrete based on a diskshaped compact tension test. J Assoc Asphalt Paving Technol 74E

15. Itasca Consulting Group, Inc (2002) PFC 2D version 3.0. Itasca Consulting Group, Inc, Minneapolis, USA

16. Cundall PA (1971) A computer model for simulating progressive, large-scale movements in blocky rock systems. In: Proceedings of the international symposium of rock fracture, Nancy, France

17. O'Sullivan C, Bray JD (2001) A comparative evaluation of two approaches to discrete element modeling of particulate media. In: Proceedings of the fourth international conference on discontinuous deformation, University of Glasgow, Scotland, UK, pp 97-110

18. Dugdale D (1960) Yielding of steel sheets containing slits. J Mech Phys Solids 8:100-104 
19. Barenblatt GI (1962) Mathematical theory of equilibrium cracks in brittle fracture. Adv Appl Mech 7:55-129

20. Hillerborg A, Modeer M, Petersson PE (1976) Analysis of crack formation and crack growth in concrete by means of fracture mechanics and finite elements. Cem Concr Res 6(6):773-782

21. Tavarez FA, Plesha ME (2006) Discrete element method for modelling solid and particulate materials. Int J Numer Methods Eng 70(4):379-404

22. Davie CT, Bićanic N (2003) Failure criteria for quasibrittle materials in lattice-type models. Commun Numer Methods Eng 19:703-713

23. Lawn B (1993) Fracture of brittle solids. Cambridge solid state science series. Cambridge University Press, Cambridge

24. Wagoner MP, Buttlar WG, Paulino HG (2005) Development of a single-edge notched beam test for asphalt concrete mixtures. J Test Eval 33(6):452-460

25. Wagoner MP (2006) Fracture tests for bituminous-aggregate mixtures: laboratory and field investigations. Ph.D. Dissertation, University of Illinois at Urbana-Champaign

26. AASHTO T322-03 (2004) Standard test method for determining the creep compliance and strength of hot mix asphalt (HMA) using the indirect tensile test device. Standard specifications for transportation materials and methods of sampling and testing, 24th edn

27. Kim H, Wagoner MP, Buttlar WG (2008) Simulation of fracture behavior in asphalt concrete using a heterogeneous cohesive zone discrete element model. ASCE J Mater Civ Eng 20(8):1-12

28. Schlangen E, Garboczi EJ (1997) Fracture simulations of concrete using lattice models: computational aspects. Eng Fract Mech 57(2/3):319-332

29. Media Cybernetics, Inc. (2004) Image-Pro Plus 5.0. Silver Spring, Maryland, USA

30. You Z (2003) Development of a micromechanical modeling approach to predict asphalt mixture stiffness using the discrete element method. Ph.D. Dissertation, University of Illinois at Urbana-Champaign

31. Tschegg EK, Stanzl-Tschegg SE, Litzka J (1993) New testing method to characterize mode I fracturing of asphalt aggregate mixture. In: Proceeding of 2nd RILEM conference, London, England, pp 263-270 